\title{
La evaluación prequirúrgica con análisis "de rutina" no previno las complicaciones perioperatorias en la cirugía de cataratas
}

The value of routine preoperative medical testing before cataract surgery. Schein OD., Katz J, Bass EB, M.D. N Engl J Med 2000; 342:168-176.

\section{Objetivo}

Determinar si la realización de análisis "de rutina" ayuda a reducir la incidencia de complicaciones médicas perioperatorias en los pacientes que van a someterse a cirugía de cataratas.

\section{Diseño}

Estudio multicéntrico aleatorizado con un seguimiento hasta una semana posterior a la cirugía.

\section{Lugar}

Nueve clínicas de EE.UU. incluyendo centros de cirugía ambulatoria, centros médicos académicos y hospitales comunitarios.

\section{Pacientes}

Se incluyeron 18.189 pacientes que se sometieron a 19.557 cirugías de cataratas (algunos fueron operados de los dos ojos). Se excluyeron los pacientes 50 años, los que requerían anestesia general, y aquellos con antecedentes de infarto de miocardio (IAM) los 3 meses previos o los que se hubieran realizado análisis dentro de los 28 días previos a la cirugía.

\section{Intervención}

Se aleatorizó a los pacientes a recibir o no evaluación prequirúrgica con estudios complementarios de rutina. A los pacientes que ingresaban al grupo "no prequirúrgico" sólo se les solicitaban análisis si presentaban alguna situación clínica que lo requiriera y cuya indicación fuera independiente de la cirugía. Los pacientes aleatorizados a l grupo " prequirúrgico" debían realizarse un electrocardiograma (ECG), hemograma completo, ionograma, urea y glucemia. A los pacientes con diabetes se les realizaba una glucemia el día de la operación independientemente del grupo al que hubieran sido aleatorizados.

\section{Medición de resultados principales}

El resultado principal fue un resultado combinado* al que se denominó "eventos médicos". Dichos eventos incluían: muerte, hospitalización, IAM, isquemia miocárdica, insuficiencia cardíaca (ICC), arritmia, hipertensión (HTA), hipotensión, accidente cerebrovascular (ACV), accidente isquémico transitorio (AIT), insuficiencia respiratoria (IR), broncoespasmo, desaturación de oxígeno, hipoglucemia, cetoacidosis diabética, coma hiperosmolar y otros.

\section{Resultados principales}

En ambos grupos (analizados por intención de tratar) la tasa acumulada de "eventos médicos" fue la misma: 31.3 por cada 1000 cirugías (RR 1 IC 95\% 0.9-1.2). Los tipos de eventos fueron similares en ambos grupos, tanto en el período operatorio como postoperatorio, a excepción del broncoespasmo que, si bien fue un evento infrecuente, fue significativamente mayor en el grupo que no recibió evaluación prequirúrgica (7 broncoespasmos vs 2). El tratamiento de HTA y arritmias (principalmente bradicardia) dieron cuenta del 61\% de los eventos en el grupo " no prequirúrgico" y $68 \%$ en el grupo " prequirúrgico" . Al evaluar por centro participante, edad, sexo o raza los resultados fueron similares. Tampoco se encontraron diferencias al analizar por enfermedades coexistentes, por la clasificación de la Asociación Americana de Anestesiología o por el estado de salud reportado por el paciente. La tasa de cancelación de cirugías fue similar en ambos grupos (2.3\% en el grupo prequirúrgico y $2.5 \%$ en el control).

\section{Conclusiones}

Realizar análisis prequirúrgico de rutina antes de la cirugía de cataratas no resulta en una disminución medible del riesgo de complicaciones perioperatorias.
Varios estudios han sugerido que la realización de extensos estudios prequirúrgicos de rutina no altera la tasa de complicaciones perioperatorias y, por lo tanto, se ha recomendado la reâlización de evaluaciones prequirúrgicas racionales acorde a la edad y la patología del paciente.1-4 Los estudios en los cuales se basan estas recomendaciones son retrospectivos. El gran valor del presente trabajo es el de haber llevado a cabo un estudio prospectivo y aleatorizado. Además está realizado en población añosa (la que tiene cataratas) y por lo tanto con mucha comorbilidad (casi 50\% de hipertensos, 20\% de anginosos, 15\% de diabéticos; por mencionar algunas de las enfermedades asociadas). Es de hacer notar que la evaluación prequirúrgica que se realizó fue bastante "acotada" si la comparamos con la que habitualmente se solicita (téngase en cuenta que no se solicitaron estudios de coagulación, por ejemplo). En todo caso, no pudo demostrarse reducción de eventos perioperatorios (a este tipo de estudio se lo llama estudio negativo, ya que no encuentra diferencias entra las ramas). Sin embargo, algunos puntos deben destacarse y ser tenidos en cuenta. Si bien el poder* del estudio para detectar diferencias entre grupos es muy alto (superior al $90 \%$ para todos los eventos combinados, calculado por EVIDENCIA), el principal problema es la combinación de tantos eventos disímiles en la misma "bolsa". Si bien el número de pacientes enrolados es más que interesante, no lo es el número de eventos que alcanzó: 301 en cada grupo. Además, los eventos no tienen igual jerarquía médica (consideremos que es un resultado combinado que combina muerte o IAM con hiper o hipotensión y que la mayoría de eventos no fueron los más graves y que, para estos eventos graves, el estudio no tuvo el poder necesario). Este empleo de un resultado combinado tan heterogéneo muestra a las claras que un estudio cuyos resultados fueran complicaciones graves o fatales sería irrealizable por el enorme número de pacientes que habría que incluir. Con relación a su validez externa*, el estudio tuvo pocas restricciones para el ingreso de los pacientes, no descartando a aquellos con comorbilidad y, por lo tanto, sus resultados son extrapolables. Tampoco hubo gran entrecruzamiento* entre ramas y el poco que hubo refuerza las conclusiones, ya que hubo más pacientes del grupo "intervención" que no la recibieron que pacientes de grupo "no intervención" que realizaron prequirúrgico. Con las salvedades antes enunciadas, este estudio aumenta el cuerpo de evidencia en favor de no realizar "prequirúrgicos de rutina" ya que solo aumenta los costos y alargan los tiempos terapéuticos, sin redundar en beneficios para los pacientes.

*Ver glosario

Dra. Karin Kopitowski Unidad de Medicina Familiar y Preventiva. Hospital Italiano de Buenos Aires.

Referencias

1. Goldberger AL et al. Utility of the routine electrocardiogram before surgery and on general admission. Critical review and guidelines. Ann Intern Med 1986; $105: 552-7$. 2. Kaplan EB, et al. The usefulness of preoperative laboratory screening. JAMA 1985; 253 (24): 3576-81.

3. Narr BJ, et al. Preoperative laboratory screening in healthy Mayo patients: cost-effectivne elimination of tests and unchanged outcomes. Mayo Clin Proc 1991; 66: 155-9. 4. Robbins JA, et al. Preoperative evaluation of the healthy patient. Med Clin North Am 1979; 63: 1145-56. 